ACTA MYCOLOGICA

Vol. 49 (1): 135-146

2014
Dedicated to Professor Maria Ławrynowicz on the occasion of the 45th anniversary of her scientific activity

DOI: $10.5586 / \mathrm{am} .2014 .010$

\title{
The preservation status of the lichen biota in the designed Special Area of Conservation NATURA 2000 „Middle Łyna River Valley - Smolajny”
}

\author{
DARIUSZ KUBIAK ${ }^{1}$, PAWEL CZARNOTA ${ }^{2}$, ANNA ZDUŃCZYK ${ }^{3}$, \\ MARIA DYNOWSKA ${ }^{1}$, GRZEGORZ LESNIANSKI ${ }^{4}$, \\ ALEKSANDRA CYGANSSKA ${ }^{5}$, SANDRA OLSZEWSKA ${ }^{6}$, \\ ANNA SADOWSKA-DES ${ }^{7}$ and PAWEL WOJDAL ${ }^{8}$
}

\author{
${ }^{1}$ Department of Mycology, Warmia and Mazury University in Olsztyn \\ Oczapowskiego 1A, PL-10-719 Olsztyn, darkub@uwm.edu.pl \\ ${ }^{2}$ Department of Agroecology, Faculty of Biology and Agriculture \\ University of Rzeszów, Ćwiklińskiej 2, PL-35-601 Rzeszów, pawczarnota@poczta.onet.pl \\ ${ }^{3}$ Department of Botany and Ecotoxycology, Institute of Biology and Environmental Protection \\ Pomeranian University in Słupsk, Arciszewskiego 22B, PL-76-200 Słupsk \\ ${ }^{4}$ Laboratory of Lichenology E3LichLab, W.M. Bartla 19C/61, PL-30-389 Kraków \\ ${ }^{5}$ Inter-Faculty Studies in Environmental Protection, University of Warsaw \\ Żwirki i Wigury 93, PL-02-089 Warsaw, grabowskaaz@wp.pl \\ ${ }^{6}$ Department of Plant Taxonomy and Nature Conservation, Gdańsk University \\ Wita Stwosza 59, PL-80-308 Gdańsk, sandra_strahl@wp.pl \\ ${ }^{7}$ Biodiversity and Climate Research Centre BiK-F, Senckenberganlage 25 \\ D-60325 Frankfurt am Main, anna-d-sadowska@o2.pl \\ ${ }^{8}$ Laboratory of Nature Protection, Faculty of Biology, University of Warsaw \\ Miecznikowa 1, PL-02-096 Warsaw, bocian.freestyle@gmail.com
}

Kubiak D., Czarnota P., Zduńczyk A., Dynowska M., Leśniański G., Cygańska A., Olszewska S., Sadowska-Deś A., Wojdal P.: The preservation status of the lichen biota in the designed Special Area of Conservation NATURA 2000 „Middle Eyna River Valley - Smolajny”. Acta Mycol. 49 (1): 135-146, 2014.

The paper presents the list of 159 taxa, including 151 lichens and 8 saprotrophic or parasitic (lichenicolous) fungi, recorded in the designed Special Area of Conservation NATURA 2000 „Middle Łyna River Valley - Smolajny” (the Forest Division of Wichrowo). The analysed area (2953 ha) covers mostly forest communities, with natural character, associated with the valley of the Łyna river (hillside lime-oak-hornbeam forests, streamside alder-ash forest, riparian black alder forest).

Key words: lichens, lichenized fungi, species diversity, forest 


\section{INTRODUCTION}

Nature conservation is one of the State Policy aspects defined in the Nature Conservation Act (Dziennik Ustaw 2004) and in the related executive regulations. One of the nature conservation objectives, within the meaning of the aforementioned act, is to preserve the biodiversity of the country. Any measures aimed at active or passive protection of species diversity require comprehensive knowledge of habitat requirements and distribution of species from different taxonomic groups. Furthermore, measures of this kind are a prerequisite for the assessment of potential threats to individual taxa. It is estimated that over $65 \%$ of biological resources in Poland are present in forests (Jaroszewicz 2007). Therefore, in view of efficient management of the Polish fauna, flora and fungi resources, the knowledge about nature resources of forest ecosystems appears to be extremely important. The State Forests are obliged by the Forest Act (Ustawa 1991) to initiate, coordinate and conduct periodic assessments of the broadly defined forest resources. In practice, this type of comprehensive assessment is usually not possible due to the lack of professionals adequately trained.

The paper presents the results of lichenological research conducted within the framework of field sessions accompanying the 25th Convention of Polish Lichenologists „Lichens in the geographic, natural and cultural space”, which was held on 6-8 September 2011 in Wichrowo Forests (N Poland, the Forest Division of Wichrowo). Due to the limited time of field work, the research focused on the most valuable and representative habitats in the study area, which include the approved or designed Special Areas of Conservation NATURA 2000. Lichens and lichenicolous fungi of Wichrowo Forests have never been investigated, and the geographical location of this forest complex and high heterogeneity of forest plant communities make the site particularly interesting in lichenological terms.

\section{STUDY AREA}

Wichrowo Forests (the Forest Division of Wichrowo) cover an area of 17645 ha and consist of two dense parts (complexes) corresponding to two subdivisions: Obręb Łaniewo and Obręb Wichrowo (Fig. 1). Each of them is situated in a different natural forest region: the forest complex of Łaniewo in the Dzielnica Elbląsko-Warmińska district of the Baltic region, whereas the complex of Wichrowo in the Dzielnica Mazursko-Podlaska region in the Pojezierze Mazurskie lakeland (Trampler et al. 1990). According to physical and geographical division proposed by Kondracki (2001), the Forest Division of Wichrowo is located in the area of four mesoregions: Pojezierze Olsztyńskie lakeland, Nizina Sępopolska lowland, Równina Ornecka plain and Wzniesienia Górowskie hills.

The land relief of Wichrowo Forests is very diverse and includes a plateau associated with the Vistulian (Baltic) glaciation. The receding glacier left behind many moraine ramparts of different directions and a dense network of dead-ice depressions transformed into peat bogs. The highest elevation in this area reaches 172 $\mathrm{m}$ a.s.l. (Kraszewo wilderness), the lowest one $-36 \mathrm{~m}$ a.s.l. (Laniewo village). The 


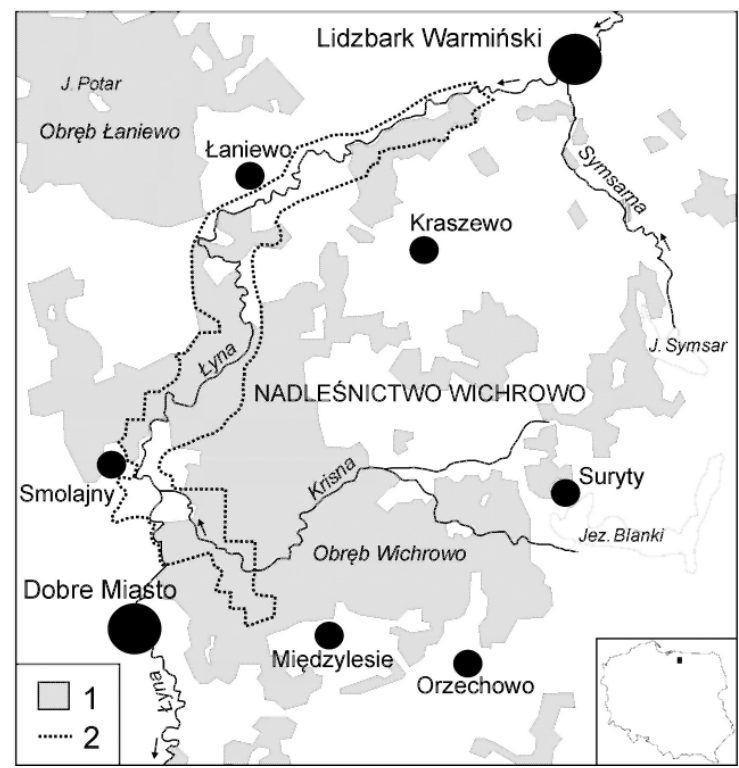

Fig. 1. Location of the analysed area in the Wichrowo Forest (the Forest Division of Wichrowo): 1 - forest areas, 2 - boundary of the designed Special Area of Conservation NATURA 2000 „Middle Łyna River Valley - Smolajny”

Forest Division area is cut through by several rivers flowing in eroded channels, which in some places developed as gorges with a relative altitude above $60 \mathrm{~m}$, and this gives the landscape typical mountain features.

Wichrowo Forests come mostly from afforestation and planting (73.1\%), and to a small extent - from natural regeneration $(2.61 \%)$ or regrowth $(3.6 \%)$ (Plan Urządzenia 2009). Scots Pine (Pinus sylvestris) is the dominant species and it covers $60.3 \%$ of the Forest Division, whereas deciduous species cover only $27.6 \%$.

The paper presents the results of the research conducted in the designed Special Area of Conservation (SAC) NATURA 2000 „Middle Łyna River Valley - Smolajny". The idea of creating SAC in Wichrowo Forests came up as a result of nature inventory carried out in the State Forests in 2006 and 2007. The area of „Middle Łyna River Valley - Smolajny" SAC includes 2953.7 ha with the Łyna Valley as a central axis (ca. $30 \mathrm{~km}$ of the river gap section between the town of Dobre Miasto and Lidzbark Warmiński) together with the estuary section of the Kirsna river flowing into Łyna (Pakulnicka et al. 2009). Forest cover $79 \%$ of the area and they are abundant in plant communities listed in the Habitat Directive, including riparian black alder forest, ash-alder forest, as well as subcontinental and hillside lime-oak-hornbeam forests. The remaining part are open areas with extensively used lowland meadows and many oxbow lakes in different developmental stages. 


\section{MATERIAL AND METHODS}

The search for species was conducted in September 2011 in two working groups at a total of 30 research sites (Tab. 1). At most sites, a complete inventory of species was made, including all ecological groups of lichens. In few cases, only rare or otherwise interesting species were found. Species identified in the field were listed without collection of herbarium specimens. In other cases - specimens were collected for further detailed taxonomic (anatomical or biochemical) analysis in a laboratory. The collected herbarium material was deposited in the following herbaria: OLTC, SLTC and GPN (Gorce National Park). The species nomenclature follows Fałtynowicz (2003) and Kirk (2013), except the genus Varicellaria (Schmitt et al. 2012). Threat categories of lichens were quoted after Cieśliński et al. (2006), and the list of lichensindicators of lowland old-growth forests - after Czyżewska and Cieśliński (2003).

\section{RESULTS}

Altogether 159 species were found in the study area, including 151 lichen species (lichenized fungi) and 8 species of saprotrophic or parasitic (lichenicolous) fungi (Tab. 2). Epiphytes dominate in the biota of lichens. Most of the species were found on oak (79 species), hornbeam (71), maple (60) and linden trees (44). The largest number of species excluded for a given phorophyte was recorded for hornbeam, oak and maple - 12 taxa each. Other ecological groups were represented by much smaller numbers: epixylic lichens - 22 species (including 12 growing exclusively on wood) and epigeic species - only 4 species. The identified lichen biota is represented by 19 taxa legally protected in Poland, including 18 strictly protected species and 1 partially protected species. Threatened lichens (facing a risk of extinction in Poland) are represented by 57 species (Tab. 2). They represent the following threat categories: critically endangered $(\mathrm{CR})-3$ species, endangered (EN, high risk of extinction) - 12, vulnerable (VU) - 21, least concerned (LC) - 3, near threatened (NT) - 16, data deficient (DD) -2 . The biota of lichens in the study area is represented by many species that have been previously known from only very few sites in the country, and their distribution, habitat preferences and potential threats are not well researched. The group includes: Bacidia hemipolia f. pallida, Biatora chrysantha, B. pontica, Biatoridium monasteriense, Fellhaneropsis vezdae, and Lecanora farinaria.

\section{DISCUSSION}

The number of lichen species reported from the study area is considered to be relatively high. It is higher than the total number of species recorded in important but small refugia of forest lichens in the southern part of the Pojezierze Olsztyńskie lakeland, including the nature reserves of „Dęby Napiwodzkie” (100 species) and Koniuszanka II" (80 species) (Kubiak 2011), but smaller than the number of species reported from the much larger forest nature reserve „Las Warmiński” - ca. 220 
Table 1

Major characteristics of the studied sites

\begin{tabular}{|c|c|c|}
\hline No. & $\begin{array}{l}\text { Location (forest section; geographical } \\
\text { coordinates) }\end{array}$ & Forest stand type (dominated tree/age) \\
\hline 1 & $403 f ; 54^{\circ} 02^{\prime} 04.2^{\prime \prime} \mathrm{N} / 20^{\circ} 23^{\prime} 36.9^{\prime \prime} \mathrm{E}$ & Oak-hornbeam forest $(Q 92)$ \\
\hline 2 & $3461 ; 54^{\circ} 02^{\prime} 44.5^{\prime \prime} \mathrm{N} / 20^{\circ} 24^{\prime} 29.0^{\prime \prime} \mathrm{E}$ & Old-growth oak forest \\
\hline 3 & $310 \mathrm{~h} ; 54^{\circ} 03^{\prime} 05.7^{\prime \prime} \mathrm{N} / 20^{\circ} 26^{\prime} 18.0^{\prime \prime} \mathrm{E}$ & Lime-oak-hornbeam forest $(Q 137)$ \\
\hline 4 & 366b; 5402'41.7”/20²4'25.7’”E & Lime-oak-hornbeam forest $(Q 192)$ \\
\hline 5 & $366 \mathrm{~b} ; 54^{\circ} 02^{\prime} 36.4^{\prime \prime} \mathrm{N} / 20^{\circ} 24^{\prime} 15.1^{\prime \prime} \mathrm{E}$ & Lime-oak-hornbeam forest $(Q 192)$ \\
\hline 6 & $347 \mathrm{i} ; 54^{\circ} 02^{\prime} 44.2^{\prime \prime} \mathrm{N} / 20^{\circ} 24^{\prime} 24.3^{\prime \prime} \mathrm{E}$ & Group of old maple trees \\
\hline 7 & $346 \mathrm{~h} ; 54^{\circ} 02^{\prime} 478^{\prime \prime} \mathrm{N} / 20^{\circ} 24 ' 35.5^{\prime \prime} \mathrm{E}$ & Group of trees at the crossroads \\
\hline 8 & $3461 ; 54^{\circ} 02^{\prime} 45.7^{\prime \prime} \mathrm{N} / 20^{\circ} 24^{\prime} 45.9^{\prime \prime} \mathrm{E}$ & $\begin{array}{l}\text { Lime-oak-hornbeam old-growth forest, hillside } \\
\text { lime-oak-hornbeam forests }(Q 242)\end{array}$ \\
\hline 9 & 291f; $54^{\circ} 03^{\prime} 40.0^{\prime \prime} \mathrm{N} / 20^{\circ} 27^{\prime} 00.2^{\prime \prime} \mathrm{E}$ & Oak forest $(Q 132)$ \\
\hline 10 & $342 \mathrm{c}(/ 343 \mathrm{a}) ; 54^{\circ} 03^{\prime} 03.5^{\prime \prime} \mathrm{N} / 20^{\circ} 26^{\prime} 07.2^{\prime \prime} \mathrm{E}$ & Lime-oak-hornbeam forest $(Q 127)$ \\
\hline 11 & $343 f ; 54^{\circ} 02^{\prime} 57.9^{\prime \prime} / 20^{\circ} 26^{\prime} 02.3^{\prime \prime E}$ & Elm-ash forest ( $A$ 52) \\
\hline 12 & $314 \mathrm{j} ; 54^{\circ} 03^{\prime} 09.6^{\prime \prime} \mathrm{N} / 20^{\circ} 25^{\prime} 34.6^{\prime \prime} \mathrm{E}$ & Mixed pine-oak forest \\
\hline 13 & $344 \mathrm{~d} ; 54^{\circ} 02^{\prime} 57.8^{\prime \prime} \mathrm{N} / 20^{\circ} 25^{\prime} 32.3^{\prime \prime} \mathrm{E}$ & $\begin{array}{l}\text { Degenerated oak-hornbeam forest (dominance } \\
\text { of hornbeam) }\end{array}$ \\
\hline 14 & $363 \mathrm{~m} ; 54^{\circ} 02^{\prime} 30.6^{\prime \prime} \mathrm{N} / 20^{\circ} 25^{\prime} 16.2^{\prime \prime} \mathrm{E}$ & Lime-hornbeam forest ( $T 75)$ \\
\hline 15 & $3630 ; 54^{\circ} 02^{\prime} 30.4^{\prime \prime} \mathrm{N} / 20^{\circ} 25^{\prime} 06.8^{\prime \prime} \mathrm{E}$ & Alder forest $(A 62)$ \\
\hline 16 & $363 \mathrm{k} ; 54^{\circ} 02^{\prime} 30.6^{\prime \prime} \mathrm{N} / 20^{\circ} 25^{\prime} 10.2^{\prime \prime} \mathrm{E}$ & Spruce forest $(\mathrm{Pa} 21)$ \\
\hline 17 & $363 \mathrm{j} ; 54^{\circ} 02^{\prime} 37.0^{\prime \prime} \mathrm{N} / 20^{\circ} 25^{\prime} 00.5^{\prime \prime} \mathrm{E}$ & Oak-hornbeam forest $(Q 102)$ \\
\hline 18 & $589 \mathrm{c} ; 53^{\circ} 59^{\prime} 43.5^{\prime \prime} \mathrm{N} / 20^{\circ} 26^{\prime} 40.0^{\prime \prime} \mathrm{E}$ & Lime-oak-hornbeam forest $(Q 107)$ \\
\hline 19 & $560 \mathrm{~g} ; 53^{\circ} 59^{\prime \prime} 59.8^{\prime \prime} \mathrm{N} / 20^{\circ} 26^{\prime \prime} 22.3 ” \mathrm{E}$ & Spruce managed forest $(Q 52)$ \\
\hline 20 & $561 \mathrm{a} ; 54^{\circ} 00 " 11.0 " \mathrm{~N} / 20^{\circ} 26 ” 01.9 ” \mathrm{E}$ & Mixed spruce-oak forest $(\mathrm{Pa} 97)$ \\
\hline 21 & $557 \mathrm{~h} ; 54^{\circ} 00^{\prime} 16^{\prime \prime} \mathrm{N} / 20^{\circ} 25^{\prime} 43.5^{\prime \prime} \mathrm{E}$ & Lime-oak-hornbeam forest $(Q 42)$ \\
\hline 22 & $528 f ; 54^{\circ} 00^{\prime} 255^{\prime \prime N} / 20^{\circ} 25^{\prime} 187^{\prime \prime} \mathrm{E}$ & Mixed spruce-pine-oak forest (PS 127) \\
\hline 23 & $560 \mathrm{k} ; 53^{\circ} 59^{\prime} 57^{\prime \prime} \mathrm{N} / 20^{\circ} 26^{\prime} 215^{\prime \prime} \mathrm{E}$ & Lime-oak-hornbeam forest $(Q 107)$ \\
\hline 24 & $589 \mathrm{a} ; 53^{\circ} 59^{\prime} 44^{\prime \prime} \mathrm{N} / 20^{\circ} 26^{\prime} 38^{\prime \prime} \mathrm{E}$ & Spruce-pine forest \\
\hline 25 & $560 \mathrm{j} ; 53^{\circ} 59^{\prime} 589^{\prime \prime} \mathrm{N} / 20^{\circ} 26^{\prime} 255^{\prime \prime} \mathrm{E}$ & Riparian black alder forest \\
\hline 26 & $197 \mathrm{~b} ; 54^{\circ} 055^{\prime} 59.4^{\prime \prime} \mathrm{N} / 20^{\circ} 29^{\prime} 53.9^{\prime \prime} \mathrm{E}$ & $\begin{array}{l}\text { Black alder forest in deep stream valley, } \\
\text { surrounded by pine forest (Ps 72) }\end{array}$ \\
\hline 27 & $196 \mathrm{~g} ; 54^{\circ} 06^{\prime} 11.1^{\prime \prime} \mathrm{N} / 20^{\circ} 29^{\prime} 57.6^{\prime \prime} \mathrm{E}$ & Mixed oak-pine forest (Ps 102) \\
\hline 28 & $185 \mathrm{c} ; 54^{\circ} 06^{\prime} 47.5^{\prime \prime} \mathrm{N} / 20^{\circ} 30^{\prime} 51.6^{\prime \prime} \mathrm{E}$ & Ash-alder forest \\
\hline 29 & $179 \mathrm{k} ; 54^{\circ} 06^{\prime} 54.9^{\prime \prime} \mathrm{N} / 20^{\circ} 31^{\prime} 24.0^{\prime \prime} \mathrm{E}$ & Oak forest $(Q 99)$ \\
\hline 30 & $309 \mathrm{~h} ; 54^{\circ} 03^{\prime} 18.5^{\prime \prime} \mathrm{N} / 20^{\circ} 26^{\prime} 34.0^{\prime \prime} \mathrm{E}$ & Lime-oak-hornbeam forest \\
\hline
\end{tabular}

Abbreviations: $A$ - Alnus, Ps - Pinus, Pa - Picea Q - Quercus, T- Tilia

Table 2

List of taxa recorded in the designed Special Area of Conservation NATURA 2000 „Middle Łyna River Valley - Smolajny”

\begin{tabular}{|l|c|c|c|}
\hline Species & Localities & Substrat & $\begin{array}{c}\text { Status of } \\
\text { the species }\end{array}$ \\
\hline Absconditella lignicola Vězda \& Pišút & 14 & $\mathrm{epx}$ & \\
\hline Acrocordia gemmata (Ach.) A. Massal. & $8,11,17,18,30$ & $C b, Q, P t, U g$ & VU \\
\hline Agonimia allobata (Stizenb.) P. James & 1 & $Q$ & \\
\hline $\begin{array}{l}\text { Amandinea punctata (Hoffm.) Coppins } \\
\text { \& Scheid. }\end{array}$ & 2,4 & $A p, C b, Q$ & \\
\hline $\begin{array}{l}\text { Anisomeridium polypori (Ellis \& } \\
\text { Everh.) M.E. Barr }\end{array}$ & $1,2,8,11,21,26$ & $A p, P t, Q, S, U g$ & \\
\hline Alyxoria varia (Pers.) Ertz \& Tehler & 4,8 & $A p$ & $\mathrm{NT}$ \\
\hline Arthonia byssacea (Weigel) Almq. & 28 & $F e$ & $\mathrm{EN}$ \\
\hline A. didyma Körb. & 4 & $C b$ & $\mathrm{EN}$ \\
\hline
\end{tabular}


Table 2 - cont.

\begin{tabular}{|c|c|c|c|}
\hline A. mediella Nyl. & 1 & $Q$ & VU \\
\hline A. radiata (Pers.) Ach. & $1,3,9,10,14,27,30$ & $C b, Q, S a$ & \\
\hline A. spadicea Leight. & $1,3,8,10,11,18,30$ & $A p, A g, C b, Q$ & \\
\hline A. vinosa Leight. & 1,11 & $A g, C b, Q$ & NT \\
\hline $\begin{array}{l}\text { Arthothelium ruanum (A. Massal.) } \\
\text { Körb. }\end{array}$ & $4,5,8,11,28$ & $\mathrm{Cb}, \mathrm{Fe}, \mathrm{Tc}$ & NT \\
\hline $\begin{array}{l}\text { *Arthrorhaphis aeruginosa R. Sant. \& } \\
\text { Tønsberg }\end{array}$ & 25 & $\begin{array}{l}\text { Cladonia coniocraeal } \\
\text { Ag }\end{array}$ & \\
\hline $\begin{array}{l}\text { Bacidia arceutina (Ach.) Rehm. \& } \\
\text { Arnold }\end{array}$ & $4,16,21,23,30$ & $\mathrm{Cb}, \mathrm{Pt}$ & EN \\
\hline B. fraxinea Lönnr. & $2,21,30$ & $C b, P t$ & DD \\
\hline $\begin{array}{l}\text { B. hemipolia f. pallida Czarnota \& } \\
\text { Coppins }\end{array}$ & $1,9,20$ & $Q$ & \\
\hline B. rubella (Hoffm.) A. Massal. & $4,9,28,30$ & $\mathrm{Ap}, \mathrm{Fe}, \mathrm{Pt}$ & VU \\
\hline B. subincompta (Nyl.) Arnold & $1,2,4-6,17,18$ & $A p, C b, Q$ & EN \\
\hline $\begin{array}{l}\text { *Bacidina chloroticula (Nyl.) Vězda } \\
\text { \& Poelt }\end{array}$ & 6 & $\begin{array}{c}\text { dead thallus of } \\
\text { Parmelia sulcatalAp }\end{array}$ & \\
\hline B. phacodes (Körb.) Vězda & 16 & $P t$ & \\
\hline $\begin{array}{l}\text { B. sulphurella (Samp.) M. Hauck \& V. } \\
\text { Wirth }\end{array}$ & $1,3,4,6,10,11,26$ & $A g, A p, C b, P a, Q$, epx & \\
\hline Biatora chrysantha (Zahlbr.) Printzen & 12 & $Q$ & \\
\hline B. efflorescens (Hedl.) Räsänen & $1-6,8,18,27,29,30$ & $A p, C b, Q, T c$ & VU \\
\hline B. globulosa (Flörke) Fr. & $1,8,12,28$ & $A p, F e, Q$ & \\
\hline B. ocelliformis (Nyl.) Arnold & 3 & $\mathrm{Cb}$ & VU \\
\hline B. pontica Printzen \& Tønsberg & 5 & $T c$ & \\
\hline $\begin{array}{l}\text { Biatoridium monasteriense J. Lahm. } \\
\text { ex Körb. }\end{array}$ & 11 & $U g$ & NT \\
\hline $\begin{array}{l}\text { Buellia griseovirens (Turner \& Borrer } \\
\text { ex Sm.) Almb. }\end{array}$ & $1-4,6,8-11,16,30$ & $A p, C b, Q, T c$ & \\
\hline Calicium adspersum Pers. & 2,4 & $Q$ & EN \\
\hline C. salicinum Pers. & 2,4 & $Q$ & VU \\
\hline C. viride Pers. & $2,4,5,7$ & $A p, Q$ & VU \\
\hline $\begin{array}{l}\text { Candelariella efflorescens R.C. Harris } \\
\& \text { W.R. Buck }\end{array}$ & 1 & $Q$ & \\
\hline C. xanthostigma (Pers ex Ach.) Lettau & 4 & $A p$ & \\
\hline Catillaria nigroclavata (Nyl.) J. Steiner & 16 & Pt & \\
\hline $\begin{array}{l}\text { Chaenotheca chlorella (Ach.) Müll. } \\
\text { Arg. }\end{array}$ & 28 & $\mathrm{Fe}$ & CR \\
\hline Ch. chrysocephala (Ach.) Th.Fr. & $1-3,5,7-9,27,28,29$ & $A p, P s, Q$ & \\
\hline Ch. ferruginea (Turner ex Sm.) Mig. & $\begin{array}{c}1-5,8,9,11,12,16 \\
28,29\end{array}$ & $A p, C b, P a, P s, Q$ & \\
\hline Ch. furfuracea (L.) Tibell & 11,21 & $\mathrm{Ag}, \mathrm{Pa}$ & NT \\
\hline Ch. stemonea (Ach.) Müll. Arg. & $2-4,29$ & $P a, Q$ & EN \\
\hline Ch. trichialis (Ach.) Hellb. & $2-5,26,28,29$ & $A g, A p, P a, Q$ & NT \\
\hline $\begin{array}{l}\text { *Chaenothecopsis savonica (Räsänen) } \\
\text { Tibell }\end{array}$ & 4 & $\begin{array}{c}C b \text { (exposed dead } \\
\text { wood })\end{array}$ & \\
\hline $\begin{array}{l}\text { Chrysothrix candelaris (L.) J.R. } \\
\text { Laundon }\end{array}$ & $4,5,7,8,28,29$ & $F e, Q$ & SP, CR \\
\hline $\begin{array}{l}\text { Cladonia chlorophaea (Flörke ex } \\
\text { Sommerf.) Spreng. }\end{array}$ & 29 & $Q$ & \\
\hline C. coniocraea (Flörke) Spreng. & $\begin{array}{c}1-5,6,9-12,16,26 \\
27,29\end{array}$ & $\begin{array}{c}A g, B p, C b, P a, P s, Q, \\
T c, \text { epx, ter }\end{array}$ & \\
\hline C. digitata (L.) Hoffm. & $4,11,27$ & Ps, epx & \\
\hline C. fimbriata (L.) Fr. & $1,9,16,26$ & $P s, Q, T c, S$ & \\
\hline C. furcata (Huds.) Schrad. & 16 & ter & \\
\hline Cliostomum corrugatum (Ach.) Fr. & 5 & $Q$ & CR \\
\hline C. griffithi (Sm.) Coppins & 1 & $Q$ & VU \\
\hline $\begin{array}{l}\text { *Clypeococcum hypocenomycis D. } \\
\text { Hawksw. }\end{array}$ & $1,2,27$ & $\begin{array}{c}\text { Hypocenomyce } \\
\text { scalaris } / P a, P s, Q\end{array}$ & \\
\hline $\begin{array}{l}\text { Coenogonium pineti (Schrad.) Lücking } \\
\& \text { Lumbsch }\end{array}$ & $\begin{array}{c}1-6,9,10,12,16,26 \\
27,29,30\end{array}$ & $\begin{array}{c}A g, A p, C b ; P a, P s, Q \\
T c, \mathrm{epx}\end{array}$ & \\
\hline
\end{tabular}


Table 2 - cont.

\begin{tabular}{|c|c|c|c|}
\hline Evernia prunastri (L.) Ach. & $1-10,12,16,28,29,30$ & $\begin{array}{c}\text { Ap, } C b, E e, F e, P a v, \\
\text { Pas, } P, Q, T c, \text { epx }\end{array}$ & PP, NT \\
\hline $\begin{array}{l}\text { Fellhanera gyrophorica Sérus., Coppins, } \\
\text { Diederich \& Scheid. }\end{array}$ & $\begin{array}{c}1,2,9,10,18,20 \\
29,30\end{array}$ & $C b, Q$ & LC \\
\hline $\begin{array}{l}\text { Fellhaneropsis myrtillicola (Erichsen) } \\
\text { Sérus. \& Coppins }\end{array}$ & 20 & $P a$ & \\
\hline $\begin{array}{l}\text { F. vezdae (Coppins \& P. James) Sérus. } \\
\text { \& Coppins }\end{array}$ & 3 & $\mathrm{Cb}$ & $\mathrm{LC}$ \\
\hline Flavoparmelia caperata (L.) Hale & 8 & $Q$ & SP, EN \\
\hline $\begin{array}{l}\text { Fuscidea arboricola Coppins \& } \\
\text { Tønsberg }\end{array}$ & $1-3,15,25$ & $A g, C b, Q$ & \\
\hline F. pusilla Tønsberg & $1,2,15,25,27$ & $A g, C b, P s, Q, T c$, ерх & \\
\hline Graphis scripta (L.) Ach. s.l. & $1-3,5,6,8-11,30$ & $\mathrm{Ap}, \mathrm{Cb}, \mathrm{Ca}, \mathrm{Tc}$ & NT \\
\hline $\begin{array}{l}\text { Hypocenomyce scalaris (Ach. ex Lijl.) } \\
\text { M. Choisy }\end{array}$ & $1,2,4,5,7,9,12,27$ & $C b, P a, P s, Q$ sp., $T c$ & \\
\hline Hypogymnia physodes (L.) Nyl. & $\begin{array}{c}1-9,11,12,15,16,26 \\
27,29,30\end{array}$ & $\begin{array}{c}A g, A p, C b, E e, P a, \\
P s, P, Q, T c, S, S a, \\
\text { epx }\end{array}$ & \\
\hline $\begin{array}{l}\text { *Ilosporiopsis christiansenii (B. L. } \\
\text { Brady \& D. Hawksw.) D. Hawksw. }\end{array}$ & 6 & $\begin{array}{l}\text { Physcia adscendens/ } \\
\text { Ap }\end{array}$ & \\
\hline Imshaugia aleurites (Ach.) S.L.F. Mey. & 7,9 & $P s, Q$ & SP \\
\hline Lecania cyrtella (Ach.) Th. Fr. & 6 & $A p$ & \\
\hline $\begin{array}{l}\text { L. naegelii (Hepp) Diederich \& van } \\
\text { den Boom }\end{array}$ & 6 & $A p$ & \\
\hline Lecanora albella (Pers.) Ach. & 4 & $A p$ & EN \\
\hline L. argentata (Ach.) Malme & $4-6,10,30$ & $A p, C b, Q$ & \\
\hline L. carpinea (L.) Vain. & $1,2,5,8,16,28,30$ & $\mathrm{Cb}, \mathrm{Fe}, \mathrm{Pt}, \mathrm{Q}$ & \\
\hline L. chlarotera Nyl. & $1-5,8-10,16,28,30$ & $A p, C b, P, Q, T c$ & \\
\hline L. compallens Herk \& Aptroot & $7,11,16$ & $\mathrm{Ag}, \mathrm{Cb}, \mathrm{Fe}, P, Q, \mathrm{Tc}$ & \\
\hline L. conizaeoides Nyl. ex Cromb. & $1,2,9$ & $C b, P a, P s, T c$ & \\
\hline L. expallens Ach. & $\begin{array}{c}1-11,14-16,26,27 \\
28,29\end{array}$ & $\begin{array}{c}A p, A g, C b, C a, F e \\
P a, Q, U g, S a, T c\end{array}$ & \\
\hline L. farinaria Borrer & 1 & $\mathrm{Cb}$ & \\
\hline L. glabrata (Ach.) Malme & $5,8,30$ & $\mathrm{Cb}$ & \\
\hline L. pulicaris (Pers.) Ach. & $1,2,4,5,9,15,16$ & $A g, C b, Q, T c$ & \\
\hline L. rugosella Zahlbr. & 16,30 & $Q, P t$ & \\
\hline L. sarcopidoides (A. Massal.) Hedl. & 24 & Ps & NT \\
\hline L. thysanophora R.C. Harris & $2,3,5,10,13,30$ & $C b, Q$ & \\
\hline Lecidea nylanderii (Anzi) Th.Fr. & 2 & $\mathrm{Cb}$ & \\
\hline $\begin{array}{l}\text { Lecidella elaeochroma (Ach.) M. } \\
\text { Choisy }\end{array}$ & $\begin{array}{c}4-6,8-10,16,26, \\
28,30\end{array}$ & $\mathrm{Ap}, \mathrm{Cb}, \mathrm{Fe}, \mathrm{Q}, \mathrm{Pt}, \mathrm{S}$ & \\
\hline $\begin{array}{l}\text { L. flavosoreditata (Vězda) Hertel \& } \\
\text { Leuckert }\end{array}$ & 4 & $A p$ & \\
\hline L. subviridis Tønsberg & $2,4,5,16,17,18$ & $A g, C b, T c$ & \\
\hline Lepraria elobata Tønsberg & $\begin{array}{c}1-6,8,9,12,13,16 \\
26,27\end{array}$ & $A g, C b, Q, P s, T c$ & \\
\hline L. incana (L.) Ach. & $1-14,16,26,27,28,29$ & $\begin{array}{c}A p, A g, B p, C b, F e, \\
P a, P s, Q, T c\end{array}$ & \\
\hline L. jackii Tønsberg & $1,2,27$ & $\mathrm{Cb}, \mathrm{Pa}, \mathrm{Ps}, \mathrm{Tc}$ & \\
\hline L. lobificans Nyl. & $1-13,26,28,29,30$ & $\begin{array}{c}A p, B p, C b, F e, Q, U g \\
T c, P t, S\end{array}$ & \\
\hline L. rigidula (B. de Lesd.) Tønsberg & $1,6,26,28$ & $\mathrm{Ag}, \mathrm{Ap}, \mathrm{Fe}, \mathrm{Tc}$ & \\
\hline L. vouauxii (Hue) R.C. Harris & 9 & $A p$ & \\
\hline $\begin{array}{l}\text { Lichenomphalia umbellifera (L.) } \\
\text { Redhead et al. }\end{array}$ & 19 & epx & NT \\
\hline $\begin{array}{l}\text { Melanohalea exasperatula (Nyl.) O. } \\
\text { Blanco et al. }\end{array}$ & 2,6 & $A p$ & SP \\
\hline $\begin{array}{l}\text { Melanelixia glabratula (Lamy) Sandler } \\
\text { \& Arup }\end{array}$ & $1-11,28,30$ & $A p, C b, P a v, Q, T c, P t$ & SP \\
\hline M. subaurifera (Nyl.) O. Blanco et al. & 8 & $P a v$ & SP \\
\hline $\begin{array}{l}\text { Micarea byssacea (Th. Fr.) Czarnota, } \\
\text { Guz.-Krzemiń. \& Coppins }\end{array}$ & 14,23 & $P t, T c$ & \\
\hline M. melaena (Nyl.) Hedl. & 15 & epk & NT \\
\hline
\end{tabular}


Table 2 - cont.

\begin{tabular}{|c|c|c|c|}
\hline $\begin{array}{l}\text { M. micrococca (Körb.) Gams ex } \\
\text { Coppins }\end{array}$ & $4,8,15,16,21$ & $F e, P s-s t C, P t, Q$, epx & \\
\hline M. misella (Nyl.) Hedl. & 22 & epx & \\
\hline $\begin{array}{l}\text { M. nitschkeana (J. Lahm. ex Rabenh.) } \\
\text { Harm. }\end{array}$ & 16 & $P t$ & \\
\hline $\begin{array}{l}\text { M. viridileprosa Coppins \& van den } \\
\text { Boom }\end{array}$ & 22 & $P S$ & \\
\hline $\begin{array}{l}\text { (*)Microcalicium dissemnatum (Ach.) } \\
\text { Vain. }\end{array}$ & 4 & $Q$ & \\
\hline $\begin{array}{l}\text { *Monodictis epilepraria Kukwa \& } \\
\text { Diederich }\end{array}$ & 1,8 & Lepraria sp./Q and $A p$ & \\
\hline $\begin{array}{l}\text { Mycobilimbia epixanthoides (Nyl.) } \\
\text { Vitik., Ahti, Kuusinen, Lommi \& T. } \\
\text { Ulvinen }\end{array}$ & $1,6,9,10,17,18,30$ & $A p, C b, Q$ & \\
\hline *Mycocalicium subtile (Pers.) Szatala & 8 & $Q$ (exposed dead & \\
\hline $\begin{array}{l}\text { *Nectriopsis rubefaciens (Ellis \& } \\
\text { Everh.) M.S. Cole \& D. Hawksw. }\end{array}$ & 15 & $\begin{array}{l}\text { Parmelia sulcatal } \\
\text { wood }\end{array}$ & \\
\hline Ochrolechia bahusiensis H. Magn. & $2,6-10$ & $A p, C b, Q, T c$ & $\mathrm{VU}^{1}$ \\
\hline O. microstictoides Räsänen & $2,5,8,30$ & $A p, C b, Q, T c$ & \\
\hline O. turneri (Sm.) Hasselrot & $4,7-9$ & $A p$, epx & \\
\hline Opegrapha rufescens Pers. & 14 & $T c$ & VU \\
\hline O. vermicellifera (Kunze) J.R. Laundon & 5,8 & $C b, T c$ & EN \\
\hline O. vulgata (Ach.) Ach. & 18 & $\mathrm{Cb}$ & VU \\
\hline Pachyphiale fagicola (Arnold) Zwackh & 26,28 & $A p, S$ & VU \\
\hline Parmelia saxatilis (L.) Ach. & $4,6,8$ & $A p, Q$ & SP \\
\hline P. sulcata Taylor & $\begin{array}{c}1-4,6,8,9,11,16,26 \\
27,28,30\end{array}$ & $\begin{array}{c}A g, A p, C b, F e, Q, \\
S, T c\end{array}$ & \\
\hline Parmeliopsis ambiqua (Wulfen) Nyl. & 9 & Ps & SP \\
\hline Peltigera didactyla (With.) J.R. Laudon & 12 & ter & SP \\
\hline P. hymenina (Ach.) Delise & 9 & epx & SP, DD \\
\hline $\begin{array}{l}\text { P. praetextata (Flörke ex Sommerf.) } \\
\text { Zopf }\end{array}$ & $8,17,18$ & $A p, Q$, epx, ter & SP, VU \\
\hline $\begin{array}{l}\text { Pertusaria albescens (Huds.) M. Choisy } \\
\& \text { Werner }\end{array}$ & $3-6,9,30$ & $A p, C b, Q, T c$ & \\
\hline P. amara (Ach.) Nyl. & $\begin{array}{c}1-3,5,7-10,12,13 \\
18,27,28,29\end{array}$ & $A p, C b, F e, Q$ & \\
\hline P. coccodes (Ach.) Nyl. & $\begin{array}{c}1-4,6,8-10,12,13 \\
16,30\end{array}$ & $A p, C b, Q, T c$ & NT \\
\hline P. coronata (Ach.) Th.Fr. & $4,5,8,16$ & $C b, Q, T c$ & VU \\
\hline P. flavida (DC.) J.R. Laundon & 4 & $\mathrm{Cb}$ & EN \\
\hline P. leioplaca DC. & $3-5,8-10,14,30$ & $A p, C b, T c$ & NT \\
\hline P. pertusa (L.) Tuck. & 3,10 & $\mathrm{Cb}$ & VU \\
\hline P. pupillaris (Nyl.) Th.Fr. & 18 & $\mathrm{Cb}$ & NT \\
\hline Phlyctis argena (Ach.) Flot. & $\begin{array}{c}1-9,11-13,16,18,26 \\
27,28,30\end{array}$ & $\begin{array}{c}A g, A p, C b, C a, F e, P, \\
P t, Q, S, T c\end{array}$ & \\
\hline Physcia adscendens (Fr.) H. Olivier & 6 & $A p$ & \\
\hline P. tenella (Scop.) DC. & 4,6 & $A p, Q$ & \\
\hline Physconia enteroxantha (Nyl.) Poelt & $1,4,6,9$ & $A p, Q$ & \\
\hline P. grisea (Lam.) Poelt & & $A p$ & \\
\hline Placynthiella dasaea (Stirt.) Tønsberg & 2,3 & epx & \\
\hline P. icmalea (Ach.) Coppins \& P. James & $2,7,15$ & epx & \\
\hline $\begin{array}{l}\text { Platismatia glauca (L.) W.L. Culb. \& } \\
\text { C.F Culb. }\end{array}$ & $2,3,5-9,11,26,27,29$ & $A g, A p, C b, P a, Q, T c$ & SP \\
\hline Porina aenea (Wallr.) Zahlbr. & $1-5,8,10,11,16,30$ & $C b, T c, Q$ & \\
\hline Pseudevernia furfuracea (L.) Zopf & 9,29 & $E e, P s$ & SP \\
\hline Psilolechia clavulifera (Nyl.) Coppins & 12 & $P a$ (exposed roots) & NT \\
\hline Pycnora sorophora (Vain.) Hafellner & 9 & Ps & \\
\hline Pyrenula nitida (Weigel) Ach. & 5,8 & $\mathrm{Cb}$ & VU \\
\hline $\begin{array}{l}\text { P. nitidella (Flörke ex Schaer.) Müll. } \\
\text { Arg. }\end{array}$ & $5,8,10$ & $\mathrm{Cb}, \mathrm{Fe}$ & EN \\
\hline
\end{tabular}


Table 2 - cont.

\begin{tabular}{|c|c|c|c|}
\hline Ramalina farinacea (L.) Ach. & $\begin{array}{c}1-3,5,6,8-10,12,16 \\
28,29,30\end{array}$ & $\begin{array}{c}A p, C b, F e, P, Q, \\
T c, P t\end{array}$ & SP, VU \\
\hline R. pollinaria (Westr.) Ach. & 2,4 & $A p, C b, Q, T c$ & SP, VU \\
\hline $\begin{array}{l}\text { Reichlingia leopoldii Diederich \& } \\
\text { Scheid. }\end{array}$ & $3,11,12$ & $A g, C b, Q, U g$ & \\
\hline Rinodina degeliana Coppins & 1 & $Q$ & \\
\hline R. efflorescens Malme & $1,2,4,6$ & $A p, C b, Q, T c$ & \\
\hline $\begin{array}{l}\text { Ropalospora viridis (Tønsberg) } \\
\text { Tønsberg }\end{array}$ & $\begin{array}{c}2,3,5,8-13,21,23 \\
26,30\end{array}$ & $\mathrm{Ag}, \mathrm{Cb}, \mathrm{Fe}, \mathrm{Pt}, \mathrm{Q}, \mathrm{Tc}$ & \\
\hline $\begin{array}{l}\text { Scoliciosporum chlorococcum (Graewe } \\
\text { ex Stenh.) Vězda }\end{array}$ & 2,8 & $C b$, epx & \\
\hline S. sarothamni (Vain.) Vězda & $2,4,6,15$ & $A g, A p, T c$ & \\
\hline $\begin{array}{l}\text { *Taeniolella punctata M.S. Christ. \& D. } \\
\text { Hawksw. }\end{array}$ & 8 & Graphis scripta/Cb & \\
\hline Thelocarpon epibolum $\mathrm{Nyl}$. & 15 & epx & $\mathrm{LC}$ \\
\hline $\begin{array}{l}\text { Trapeliopsis flexuosa (Fr.) Coppins \& } \\
\text { P. James }\end{array}$ & 3,7 & epx & \\
\hline T. granulosa (Hoffm.) Lumbsch & $2,4,9$ & epx & \\
\hline Usnea filipendula Stirt. & 7,29 & $Q$ & SP, VU \\
\hline U. subfloridana Stirt. & 9,29 & $C b, Q$ & SP, EN \\
\hline $\begin{array}{l}\text { Varicellaria hemisphaerica (Florke) } \\
\text { Schmitt \& Lumbsch }\end{array}$ & $3,5,6,7,10$ & $A p, Q, T c$ & VU \\
\hline Violella fucata (Stirt.) T. Sprib. & $1,2,4-6,10,15,27$ & $A p, A g, C b, T c, S a$ & \\
\hline $\begin{array}{l}\text { Vulpicida pinastri (Scop.) J.-E. } \\
\text { Mattsson }\end{array}$ & 15 & $A g$ & SP, NT \\
\hline Xanthoria parietina (L.) Beltr. & 6 & $A p$ & \\
\hline X. polycarpa (Hoffm.) Rieber & 6 & $A p$ & \\
\hline $\begin{array}{l}\text { Zwackhia viridis (Ach.) Poetsch \& } \\
\text { Schied. }\end{array}$ & $2-6,8-11$ & $A p, A g, C b, Q$ & VU \\
\hline
\end{tabular}

Abbreviations: * - saprotrophic or parasitic (lichenicolous) fungus; $A g$ - Alnus glutinosa, Ap - Acer platanoides, $\mathrm{Bp}$-Betula pendula, $\mathrm{Ca}$-Corylus avellana, $\mathrm{Cb}$-Carpinus betulus, Ee-Euonymus europaea, $\mathrm{Fe}$ - Fraxinus excelsior, Pa - Picea abies, Pav - Padus avium, Ps - Pinus sylvestris, $P$ - Populus sp., Pt - Populus tremula, $Q$ - Quercus sp., $S$ - Salix sp., Sa - Sorbus auccuparia, Tc - Tilia cordata; ter - terricolous, epx epixylic; $\mathrm{SP}$ - strictly protected species, $\mathrm{PP}$ - partially protected species; $\mathrm{CR}$ - critically endangered, EN - endangered, VU - vulnerable, NT - near threatened, LC - least concern, DD - data deficient; ${ }^{1}$ - as $O$. androgyna s.l.

species (Kubiak 2012). So far pine forest habitats with the sites of terricolous lichens and non-forest habitats - e.g., roadside woodlots, have been investigated to a small extent only. Furthermore, the data on lichenicolous fungi should be regarded as a contribution to the knowledge about the group.

It appears that the species diversity of lichens in the most valuable fragments of the study area, including meso- and eutrophic communities of oak-hornbeam forests, riverine forests and alder swamp woods, is well evidenced by the conducted research. Mixed lime-oak-hornbeam forests are one of the most valuable plant communities in terms of lichen biota heterogeneity. Many stenotopic lichens occur at these sites, in particular epiphytes and epixylites (Cieśliński et al. 1995; Czyżewska 2003). Although there are relatively many thematically diverse papers on lime-oakhornbeam forests, the contribution of lichens in these communities is not thoroughly explored and lichens are basically overlooked in phytosociological studies.

Biodiversity of forest ecosystems largely depends on their natural conditions (Jaroszewicz 2007) and in the case of organisms like lichens, this is a determining factor (Cieśliński, Tobolewski 1988; Cieśliński et al. 1996; Czyżewska, Cieśliński 2003; Kubiak, Sucharzewska 2012). Species that are natural components of forest biocenoses dominate in the lichen biota of the study area. Stenotopic lichens are well 
represented - they are less and less frequent outside the area of extensive and well preserved forests. When comparing the species composition of lichens recorded in the Eyna Valley with the results obtained in the strongly deforested, agricultural area of the Równina Warmińska plain (Szymczyk, Zalewska 2008), the number of lichen species growing in Wichrowo Forests is much larger (Równina Warmińska plain - 96 species, the Łyna Valley - 152). There were only 44 lichen species common for both compared areas - mostly widespread, ubiquitous and hemerophilous. Although the number of protected species recorded in the Równina Warmińska plain is similar 14 species (the Łyna Valley - 19), threatened species were much fewer - 18 species (the Łyna Valley - 57).

The relatively large number of lichens attached to the bark of old oak trees, rare or even very seldom species, threatened with extinction in Poland, is one of the most valuable components of the lichen biota, and as it appears - differential for the studied fragment of Wichrowo Forests. The group includes: Calicium adspersum, C. viride, Chrysothrix candelaris, Cliostomum corrugatum i Microcalicium disseminatum. In Poland, these taxa are regarded as indicators of lowland old-growth forests (Czyżewska, Cieśliński 2003). A total of 21 species with this status were found in the study area (tab. 2). This number is relatively large compared to previously researched and described, similar refugia of forest lichens (Czyżewska, Cieśliński 2003). Some of the listed species occur in the study area with a relatively high frequency (eg., Chrysothrix candelaris, Fellhanera gyrophorica, Varicellaria hemisphaerica, Zwackhia viridis) compared to forests outside the nature reserves.

The occurrence of many lichen species in forests is determined by high species and age diversity of forest stands, owing to which individual taxa make use of the evolutionary consolidated ecological amplitude in relation to substrate (tree species), and consequently, may exist at a given site (see Cieśliński 2008). Oak is of particular significance in this respect, because of the longevity and diverse structure of periderm varying with age (Cieśliński 2008; Kubiak, Sucharzewska 2012). A total of 79 species of lichens and saprotrophic fungi were found on the bark of this phorophyte in the study area, which is a relatively large number (Kubiak 2011) and is higher than the number of species recorded on the bark of oak trees in the forest reserves „Dęby Napiwodzkie” and „Koniuszanka II” (71 species) with unique oldgrowth oak forest (Kubiak 2011). Many authors emphasize the importance of old oak trees for the preservation of species richness epiphytic lichens in forests, both in quantitative and qualitative terms (Cieśliński 2008; Johansson et al. 2009; Kubiak, Zalewska 2009; Kubiak, Sucharzewska 2012).

\section{SUMMARY}

As evidenced by the results presented and compared to other forests in Warmia and Masuria not included yet in the nature reserve conservation programme, the analysed fragment of Wichrowo Forests is characterised by above-average lichenological values and deserve protection. Due to the unique geographical location and land relief, the area functions as an ecological corridor and is potentially very valuable in the nature conservation system of the Warmia-Masuria province. Although 
after verification, the area was ultimately removed from the list of potential Sites of Community Importance (SCI), its natural values are high and fully deserve detailed exploration and protection. Due to the limited scope of the research conducted, further lichenological research in Wichrowo Forests should be continued, both in other types of forest plant communities and in non-forest habitats directly connected with a forest.

Acknowledgements. The authors are very grateful to Mr. Andrzej Reguła (the Forest Division Manager) and other employees of the Wichrowo Forest Inspectorate for their comprehensive assistance during the field work, and to the anonymous reviewers for helpful remarks on the manuscript.

\section{REFERENCES}

Cieśliński S. 2003. Atlas rozmieszczenia porostów (Lichenes) w Polsce Północno-Wschodniej. Phytocoenosis (N.S.) 15, Suppl. Cartogr. Geobot. 15: 1-430.

Cieśliński S. 2008. Znaczenie ochrony rezerwatowej dla zachowania bioty porostów (Ascomycota lichenisati) w Puszczy Kozienickiej. Stud. i Mat. CEPL, Rogów 10, 3 (19): 99-109.

Cieśliński S., Czyżewska K. 1992. Problemy zagrożenia porostów w Polsce. Wiad. Bot. 36 (1-2): 5-17.

Cieśliński S., Czyżewska K. 2003. Czerwona lista porostów wymarłych i zagrożonych w Polsce. Monogr. Bot. 91: 13-49.

Cieśliński S., Czyżewska K., Fabiszewski J. 2006. Red list of the lichens in Poland. (In:) Z. Mirek, K. Zarzycki, W. Wojewoda, Z. Szeląg (eds). Red list of plants and fungi in Poland. W. Szafer Institute of Botany, Polish Academy of Sciences, Kraków: 71-89.

Cieśliński S., Czyżewska K., Faliński J. B., Klama H., Mułenko W., Żarnowiec J. 1996. Relicts of the primeval (virgin) forest. Relict phenomena. (In:) J.B. Faliński, W. Mułenko (eds). Cryptogamous plants in the forest communities of Białowieża National Park (Project CRYPTO 3). Phytocoenosis 8 (N.S.), Archivum Geobot. 6: 197-216.

Cieśliński S., Czyżewska K., Glanc K. 1995. Lichenes. (In:) J.B. Faliński, W. Mułenko (eds). Cryptogamous plants in the forests communities of Białowieża National Park. General problems and taxonomic groups analysis (Project CRYPTO). Phytocoenosis 7 (N.S.), Archiv. Geobot. 4: 75-86.

Cieśliński S., Fałtynowicz W. 1993. Note from editors. (In:) S. Cieśliński, W. Fałtynowicz (eds). Atlas of the geographical distribution of lichens in Poland 1: 7-8. W. Szafer Institute of Botany, Polish Academy of Sciences, Kraków.

Cieśliński S., Tobolewski Z. 1988. Porosty (Lichenes) Puszczy Białowieskiej i jej zachodniego przedpola. Phytocoenosis 1 (N.S.), Suppl., Cartograph. Geobot. 1: 3-216.

Czyżewska K. 2003. Ocena zagrożenia bioty porostów Polski. Monogr. Bot. 91: 241-249.

Czyżewska K., Cieśliński S. 2003. Porosty - wskaźniki niżowych lasów puszczańskich w Polsce. Monogr. Bot. 91: 223-239.

Fałtynowicz W. 2003. The lichens, lichenicolous and allied fungi of Poland. An annotated checklist. W. Szafer Institute of Botany, Polish Academy of Sciences, Kraków.

Jaroszewicz B. 2007. Różnorodność biologiczna lasów polskich. Wszechświat 108 (46): 216-221.

Johansson V., Bergman K., O., Lättman H., Milberg P. 2009. Tree and site quality references of six epiphytic lichens growing on oaks in southeastern Sweden. Ann. Bot. Fenn. 46: 496-506.

Kirk P.M. 2013. Species Fungorum. Digital resources at www.speciesfungorum.org/Names/Names.asp. Accessed 15 April 2013.

Kondracki J. 2001. Geografia regionalna Polski. PWN, Warszawa.

Kubiak D. 2011. Stan zachowania bioty porostów w rezerwatach „Dęby Napiwodzkie” i „Koniuszanka II” na Pojezierzu Olsztyńskim. Parki nar. Rez. Przyr. 30 (3-4): 27-39.

Kubiak D. 2012. Protected and threatened lichen species of the Nidzica Primeval Forest (N Poland). (In:) L. Lipnicki (ed.). Lichen protection - Protected lichen species. . Sonar Literacki, Gorzów Wlkp.: 263-276.

Kubiak D., Sucharzewska E. 2012. Porosty - wskaźniki niżowych lasów puszczańskich w zespołach leśnych rezerwatu „Las Warmiński” (Nadleśnictwo Nowe Ramuki). Sylwan 156 (8): 627-636. 
Kubiak D., Zalewska A. 2009. Notes on Caloplaca lucifuga (Teloschistales, Ascomycota) in Poland. Acta Mycol. 44 (2): 239-248.

Pakulnicka J., Górski A., Lewandowski K., Kruszelnicki J. 2009. Dolina Środkowej Łyny - Smolajny. (In:) W: Hołdyński C., Krupa M. (eds). Obszary NATURA 2000 w województwie warmińsko-mazurskim: 137-140. Wydawnictwo Mantis, Olsztyn.

Plan Urządzenia Lasu na lata 2009-2018. Nadleśnictwo Wichrowo, Obręb Wichrowo. Program Ochrony Przyrody. Stan na 1.01.2009 r. Biuro Urządzania Lasu i Geodezji Leśnej Oddział w Olsztynie.

Schmitt I., Otte J., Parnmen S., Sadowska-Deś A.D., Lucking R., Lumbsch H.T. 2012. A new circumscription of the genus Varicellaria (Pertusariales, Ascomycota). MycoKeys 4: 23-36.

Szymczyk R., Zalewska A. Lichens in the rural landscape of the Warmia Plain. Acta Mycol. 43 (2): 215230.

Trampler T., Kliczkowska A., Dmytreko E., Sierpińska A. 1990. Regionalizacja przyrodniczo-leśna na podstawach ekologiczno-fizjograficznych. PWRiL, Warszawa.

Ustawa z dnia 28 września 1991 r. o lasach Dz.U. 1991 Nr 101 poz. 444 (z późniejszymi nowelizacjami).

Ustawa z dnia 16 kwietnia 2004 r. o ochronie przyrody Dz.U. 2004 nr 92 poz. 880.

\title{
Stan zachowania bioty porostów projektowanego Specjalnego Obszaru Ochrony Siedlisk NATURA 2000 „Dolina Środkowej Łyny - Smolajny”
}

\begin{abstract}
Streszczenie
Praca przedstawia wyniki badań lichenologicznych przeprowadzonych w dniach 6-8 września 2011 r. w ramach sesji terenowej towarzyszącej 25 Zjazdowi Lichenologów Polskich, który odbywał się w Medynach koło Lidzbarka Warmińskiego. Celem sesji było poznanie zasobów gatunkowych porostów położonych na terenie Lasów Wichrowskich (Nadleśnictwo Wichrowo), istniejących lub projektowanych, Specjalnych Obszarów Ochrony Siedlisk (SOOS) Natura 2000. W tej pracy przedstawiono wyniki inwentaryzacji przeprowadzonej na obszarze zaproponowanego do utworzenia SOOS „Dolina Środkowej Łyny - Smolajny”. Obszar o powierzchni 2953 ha obejmuje głównie zbiorowiska leśne, związane z przełomowym odcinkiem rzeki Łyny (grądy typowe, grądy zboczowe, łęgi, olsy). Na analizowanym terenie znaleziono 159 taksonów, w tym 151 gatunków porostów (grzybów zlichenizowanych) oraz 8 gatunków grzybów saprotroficznych lub pasożytniczych (naporostowych). Biota ta obejmuje 19 taksonów objętych w Polsce ochroną oraz 57 zagrożonych w skali kraju wymarciem. Ponadto, stwierdzono gatunki znane dotychczas w kraju z bardzo nielicznych stanowisk, do których można zaliczyć: Bacidia hemipolia f. pallida, Biatora chrysantha, B. pontica, Biatoridium monasteriense, Fellhaneropsis vezdae, Lecanora farinaria. Na podstawie uzyskanych wyników można stwierdzić, że analizowany fragment Lasów Wichrowskich, na tle innych kompleksów leśnych Warmii i Mazur nie objętych dotychczas ochroną rezerwatową, wykazuje ponadprzeciętne wartości lichenologiczne, które zasługują na ochronę.
\end{abstract}

